\title{
Multimodal imaging of late-onset retinal degeneration complicated by bilateral choroidal neovascularization
}

\author{
Nakul Mandal $^{1} \cdot$ Andrew J. Lotery $\mathbb{( D}^{1}$ \\ Received: 5 January 2019 / Accepted: 15 January 2019 / Published online: 28 January 2019 \\ (c) The Royal College of Ophthalmologists 2019
}

We report on the management of a case of bilateral macular choroidal neovascular membranes (CNVMs) associated with late-onset retinal degeneration (LORD).

A 54-year old woman presented with a 6-year history of nyctalopia. Both her father and paternal grandfather had a history of night blindness. Snellen visual acuities were 6/6 OD and $6 / 5$ OS. Fundal examination revealed bilateral macular atrophy and drusen-like deposits, with well-defined scalloped areas of RPE atrophy in the mid-periphery. Cone mediated electroretinography (ERG) was normal, however rod and mixed photoreceptor responses were significantly below normal levels in both eyes. Furthermore, pattern ERG and occipital pattern visual evoked potentials to the smallest $10^{\prime}$ checks were degraded, indicative of reduced bilateral macular function.

At the planned 6-month follow-up no change in the vision had been noticed by the patient though visual acuities were found to be $6 / 7.5$ OD and 6/24 OS. Examination revealed a left CNVM with subretinal fluid and a course of three ranibizumab (Lucentis) intravitreal injections at monthly interval were initiated (Fig. 1a, b). Subsequent DNA analysis identified a heterozygous C1QTNF5 change c. $489 \mathrm{C}>\mathrm{G}$ p.(Ser163Arg), confirming the diagnosis of LORD.

Visual acuity was 6/7.5 OD and 6/15 OS at 1 month following the third ranibizumab injection. Examination revealed no fluid in the left macula, however, an extrafoveal CNVM with mild subretinal fluid had now developed in the right macula. A course of three bevacizumab (Avastin) intravitreal injections at one-month interval was commenced in the right eye. At 6 week follow up visual acuity

Andrew J. Lotery

a.j.lotery@soton.ac.uk

1 Clinical and Experimental Sciences, Faculty of Medicine, University of Southampton, and Eye Unit, University Hospital Southampton NHS Foundation Trust, Southampton, UK was 6/7.5 OD and 6/24 OS with examination showing no macular fluid bilaterally, which remained the case over the following months (Fig. 1c, d). After a further 17 months, visual acuities were 6/38 OD and 6/24 OS with OCT examination revealing intra- and subretinal fluid bilaterally (Fig. 1e-h). A further course of three intravitreal bevacizumab injections at monthly interval were initiated in both eyes, with plan for continued close monitoring and treatment.

\section{Discussion}

LORD is an autosomal dominant ocular disease involving a mutation in the C1QTNF5 gene on chromosome 11, encoding Complement Component 1q and Tumor Necrosis Factor Related Protein 5. This rare condition is characterized by the onset of nyctalopia in midlife with progressive decline in central and peripheral vision. Fundus features include drusenoid deposits, pigmentary retinopathy, chorioretinal atrophy, CNVM, and retina-wide thick extracellular deposition between the retinal pigment epithelium and Bruch's membrane [1, 2]. Indeed, LORD may be mistaken for a number of conditions such as Sorsby fundus dystrophy, choroideraemia, retinitis pigmentosa, and agerelated macular degeneration. Our patient also showed the typical features of peripupillary iris atrophy and long anteriorly inserted lens zonules, which if present may aid diagnosis prior to genetic testing (Fig. 2).

Some studies have shown a positive effect of vitamin A supplementation in slowing the progression of LORD, whilst laser photocoagulation of associated CNVM has not been promising [3]. We have demonstrated that such CNV lesions are responsive to anti-vascular endothelial growth factor therapy.

Within the eye, C1QTNF5 is significantly expressed in the RPE, ciliary process and lens epithelium, with studies showing an important role in adhesion between the RPE and Bruch's membrane $[2,3]$. Continued research is necessary to 
further elucidate the function of C1QTNF5 and the pathogenesis of LORD, which could lead to the development of novel treatment strategies, possibly including retinal gene (CRISPR/Cas9) and cell based therapies [4-6].

\section{Compliance with ethical standards}

Conflict of interest The authors declare that they have no conflict of interest.
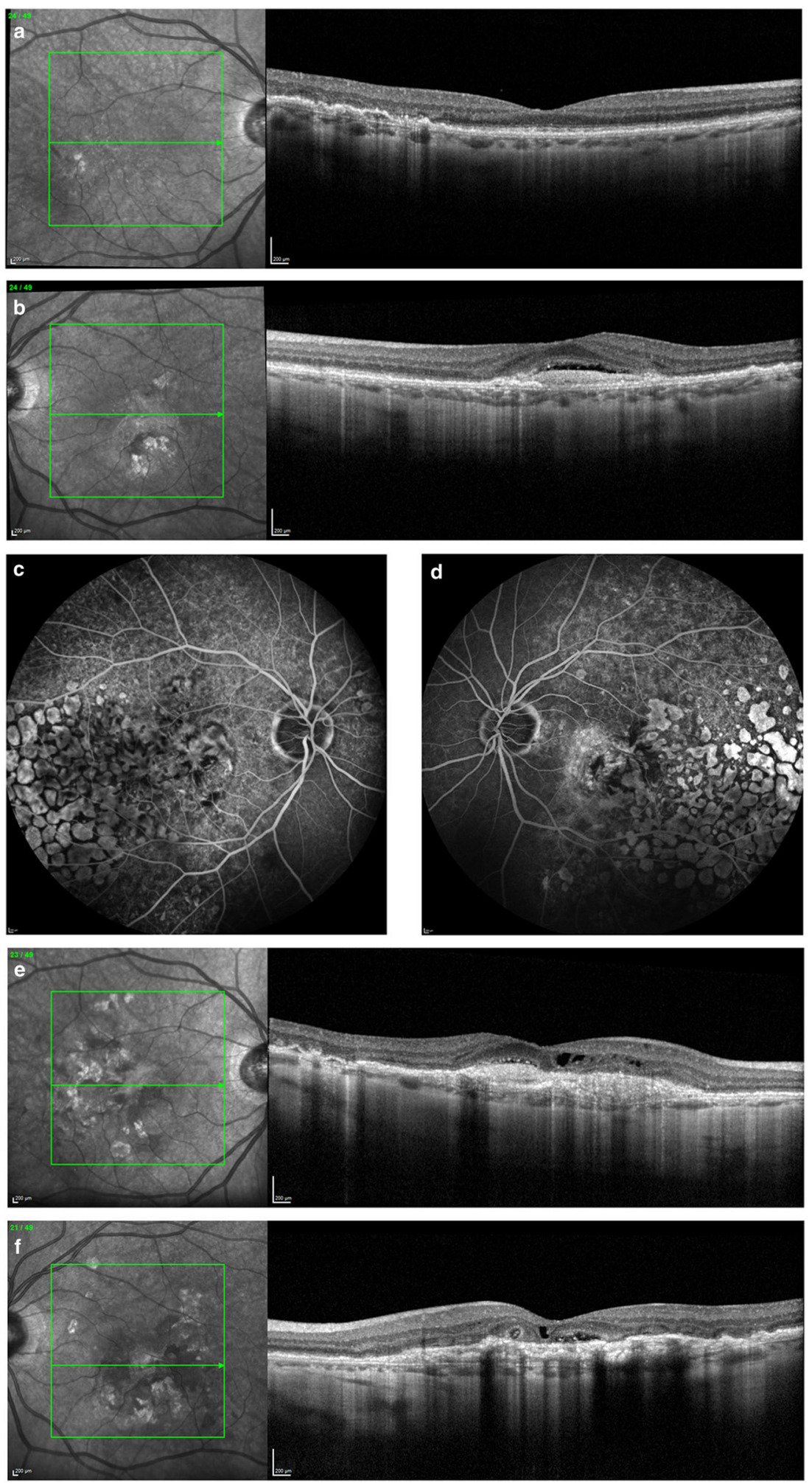

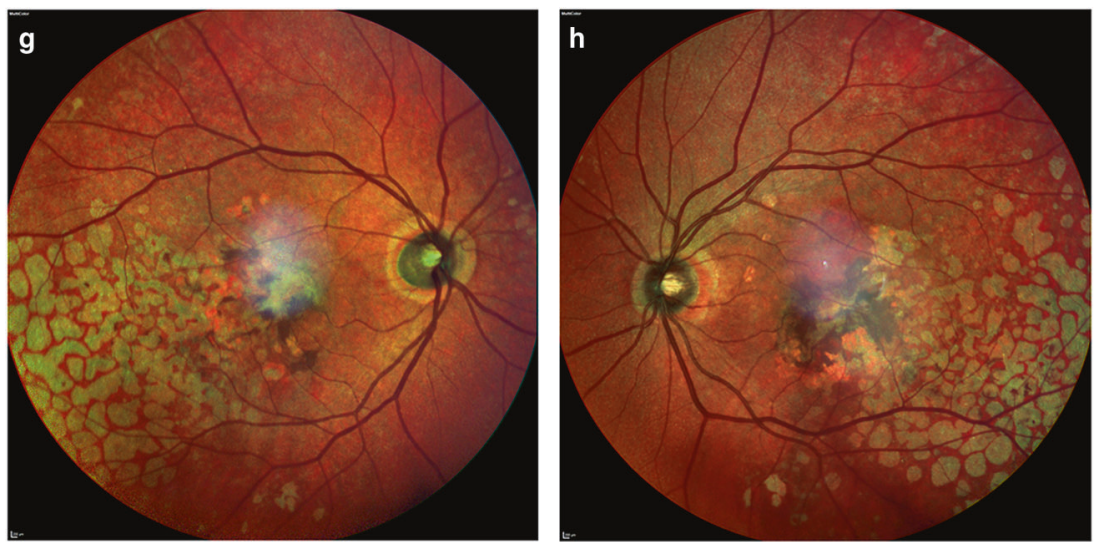

Fig. 1 LORD with secondary CNVM imaged by Heidelberg Spectralis optical coherence tomography (a, b, e, f), MultiColor (g, h), and fluorescein angiography $(\mathbf{c}, \mathbf{d})$

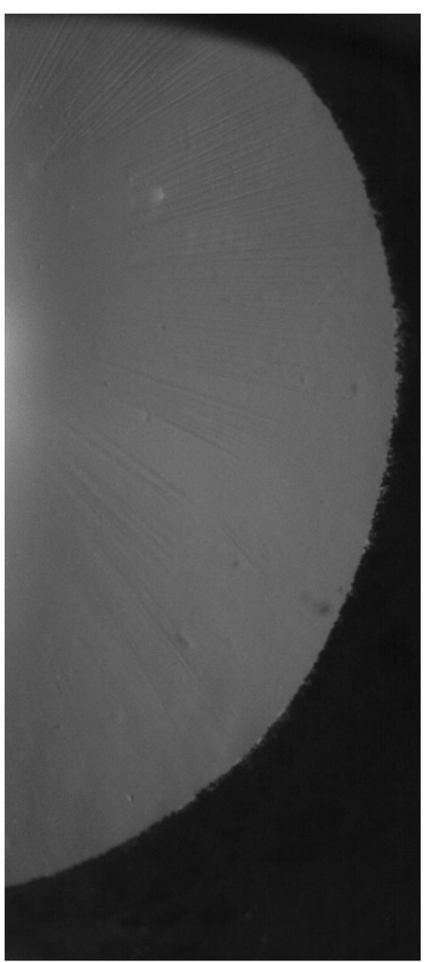

Fig. 2 Anterior segment photo showing peripupillary iris atrophy and long anteriorly inserted lens zonules
Publisher's note: Springer Nature remains neutral with regard to jurisdictional claims in published maps and institutional affiliations.

\section{References}

1. Kuntz CA, Jacobson SG, Cideciyan AV, Li ZY, Stone EM, Possin D, et al. Sub-retinal pigment epithelial deposits in a dominant late-onset retinal degeneration. Invest Ophthalmol Vis Sci. 1996;37:1772-82.

2. Hayward C, Shu X, Cideciyan AV, Lennon A, Barran P, Zareparsi $S$, et al. Mutation in a short-chain collagen gene, CTRP5, results in extracellular deposit formation in late-onset retinal degeneration: a genetic model for age-related macular degeneration. Hum Mol Genet. 2003;12:2657-67.

3. Borooah S, Collins C, Wright A, Dhillon B. Late-onset retinal macular degeneration: clinical insights into an inherited retinal degeneration. Br J Ophthalmol. 2009;93:284-9.

4. Xue K, Jolly JK, Barnard AR, Rudenko A, Salvetti AP, Patricio $\mathrm{MI}$, et al. Beneficial effects on vision in patients undergoing retinal gene therapy for choroideremia. Nat Med. 2018;24:1507-12.

5. da Cruz L, Fynes K, Georgiadis O, Kerby J, Luo YH, Ahmado A, et al. Phase 1 clinical study of an embryonic stem cell-derived retinal pigment epithelium patch in age-related macular degeneration. Nat Biotechnol. 2018;36:328-37.

6. Stanton CM, Borooah S, Drake C, Marsh JA, Campbell S, Lennon A, et al. Novel pathogenic mutations in C1QTNF5 support a dominant negative disease mechanism in late-onset retinal degeneration. Sci Rep. 2017;7:12147. 


\title{
Visual loss secondary to fludarabine toxicity: optical coherence tomography findings in two patients
}

\author{
Jonathan Virgo $^{1} \cdot$ Graeme Williams $^{2} \cdot$ Miles Stanford $^{1}$ \\ Received: 19 December 2018 / Accepted: 7 January 2019 / Published online: 28 January 2019 \\ (c) The Royal College of Ophthalmologists 2019
}

Fludarabine is a purine analogue used to treat haematological malignancy and for conditioning prior to allogenic bone marrow transplant. In early clinical trials [1] high-dose fludarabine caused high rates of severe neurotoxicity $(56 \%)$ and death $(44 \%)$. Fludarabine is now used at lower doses in combination with other chemotherapy drugs (e.g. FLAG-Ida regimen). However, the risk of severe sight loss and blindness in one study [2] was $1 \%$ and $0.3 \%$, respectively.
We present two patients with painless loss of vision secondary to fludarabine toxicity. Both had normal ophthalmic examination and optical coherance tomography (OCT) at presentation. Patient 1 developed blurred vision 14 days after FLAG-Ida cycle 2. At presentation visual acuities (VA) bilaterally were $6 / 18$. Six weeks later VA worsened to $6 / 36$ and high-dose oral prednisolone was prescribed. Initially VA improved to $6 / 9$ bilaterally, but declined to $6 / 60$ over 3 years.

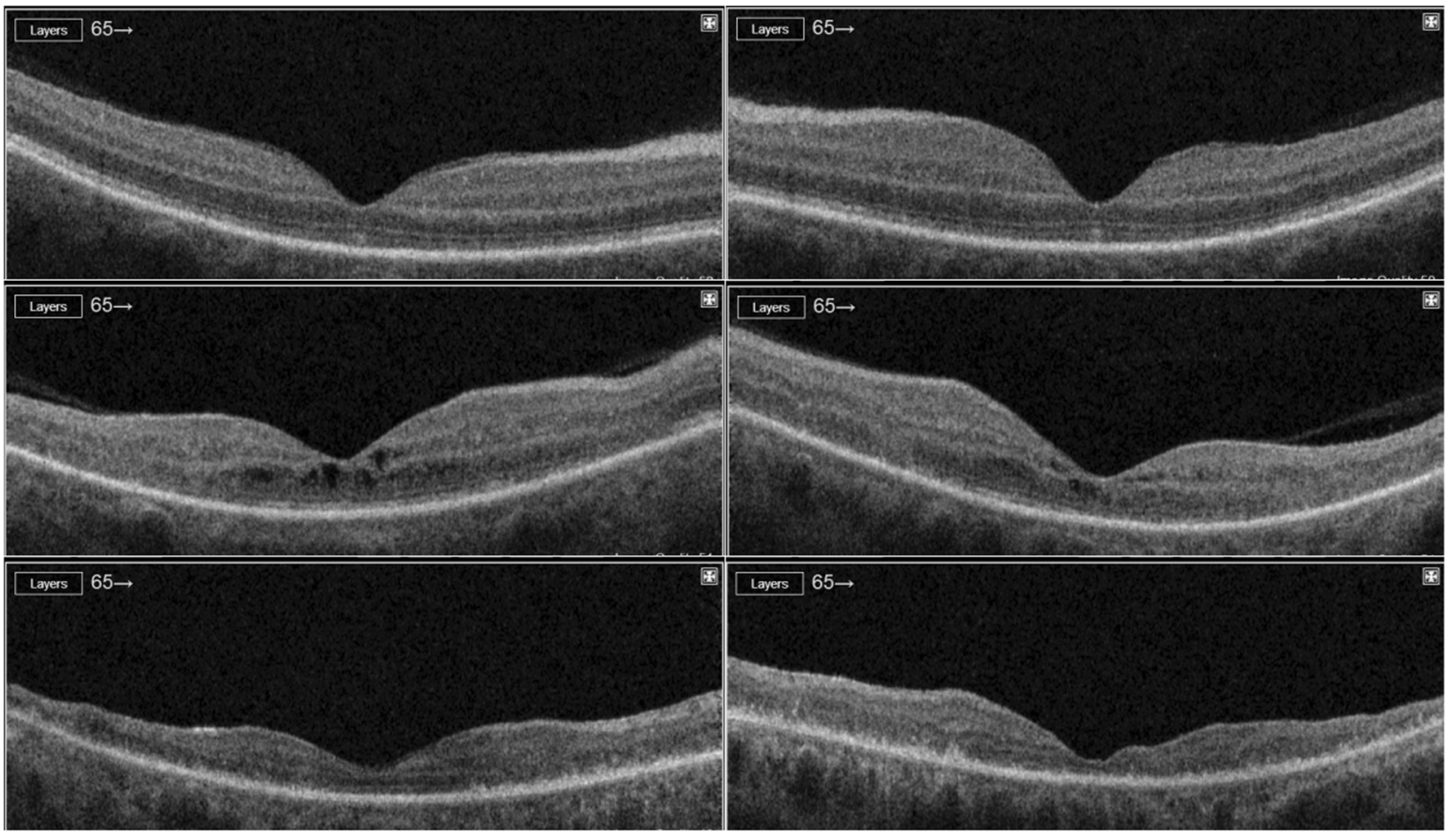

Fig. 1 Macular optical coherence tomography from patient 1 at 30 days post chemotherapy (top), 10 months post chemotherapy (middle) and 3 years post chemotherapy (bottom)

Jonathan Virgo

jdvirgo@nhs.net
Medical Eye Unit, St Thomas' Hospital, London SE1 7EH, UK

2 Ophthalmology Department, Gartnavel General Hospital, Glasgow G12 0YN, UK 
Patient 2 experienced dimming of vision during FLAG-Ida cycle 1, with VA 6/9 bilaterally. Despite a healthy ocular surface, keratoconjunctivitis secondary to cytarabine was diagnosed and the dose halved. During cycle 2 VA dropped to perception of light in both eyes, but recovered in $<1$ week to 6/9 with high-dose oral prednisolone and remained stable throughout 8 months' follow-up.

Punctate yellow intraretinal macular flecks, previously reported with fludarabine toxicity [3], were not observed in our patients. OCT features have not previously been reported. Electronegative electroretinogram (ERG) and bipolar cell dysfunction are previously reported findings $[3,4]$. Histopathology shows loss of retinal ganglion cells, loss of bipolar cells and inner nuclear layer oedema; inner retina and optic nerve head gliosis and inflammatory cell infiltrate are also reported [3, 4]. Fludarabine can also cause brain white matter toxicity [5], affecting the occipital lobes with central visual loss.

Initially, bright flash rod ERGs from patient 1 were electronegative with reduced oscillatory potentials (OPs), indicating inner retina dysfunction. Over time the B wave improved, but OPs did not. Multifocal ERGs were globally reduced and delayed, and worsened over 2 years. OCTs, normal at presentation, over time showed microcystic macular oedema (inner nuclear layer oedema), outer nuclear layer oedema and photoreceptor layer disruption involving the external limiting membrane and ellipsoid zone, along with generalised retinal thinning (Fig. 1).

Bright flash rod ERGs from patient 2 were also electronegative. Pattern ERGs showed a low N95/P50 ratio suggesting retinal ganglion cell dysfunction. OCTs, normal at presentation, developed microcystic macular oedema, outer
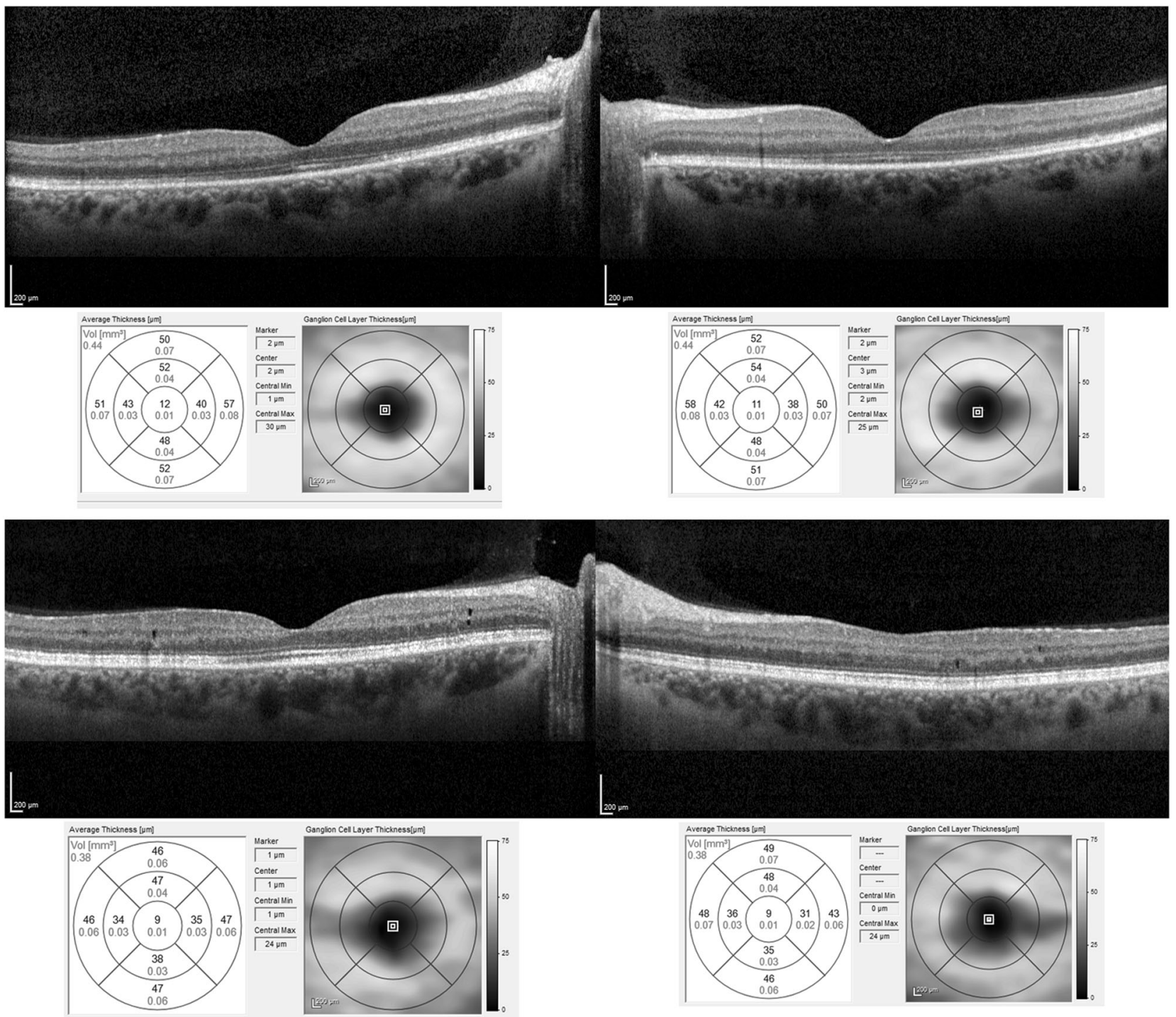

Fig. 2 Macular optical coherence tomography from patient 2 at 4 days post chemotherapy (top two rows) and 8 months post chemotherapy (bottom two rows) 
nuclear layer oedema and thinning of the ganglion cell layer during follow-up (Fig. 2). At presentation magnetic resonance imaging (MRI) showed subtle white matter changes in the occipital lobes.

Fludarabine-related visual loss is well documented and our patients had several classic features. Diagnosis must be made based on history, as initial examination and OCT are normal. Fludarabine must be stopped when toxicity is suspected and investigated with ERG and brain MRI. This report is the first to include OCT. Serial OCTs during follow-up show the evolution of various retinal changes (see above), most of which have previously been shown via histopathology in case reports. There is currently no good quality evidence to guide treatment.

\section{Compliance with ethical standards}

Conflict of interest The authors declare that they have no conflict of interest.
Publisher's note: Springer Nature remains neutral with regard to jurisdictional claims in published maps and institutional affiliations.

\section{References}

1. Warren RP, Jr, Berman E. Phase I and II study of fludarabine phosphate in leukemia: therapeutic efficacy with delayed central nervous system toxicity. J Clin Oncol. 1986;4:74-9.

2. Sorensen, et al. Treatment of refractory chronic lymphocytic leukemia with fludarabine phosphate via the Group C Protocol Mechanism of the National Cancer Institute: five-year follow-up report. J Clin Oncol. 1997;15:458-65.

3. Bishop, et al. Rapid vision loss associated with fludarabine administration. Retina. 2010;30:1272-7.

4. Ding, et al. Ocular toxicity of fludarabine: a purine analog. Expert Rev Ophthalmol. 2008;3:97-109.

5. Lee, et al. Clinical and Imaging Features of Fludarabine Neurotoxicity. J Neuroophthalmol. 2010;30:37-41.

\title{
Needles as a source of silicone oil during intravitreal injection
}

\author{
Gustavo Barreto Melo $\mathbb{1}^{1,2} \cdot$ Geoffrey Guy Emerson ${ }^{3} \cdot$ Acácio Alves Souza Lima Filho ${ }^{2} \cdot$ Shoko Ota ${ }^{4}$ Maurício Maia ${ }^{2}$
}

Received: 20 December 2018 / Accepted: 21 December 2018 / Published online: 13 February 2019

(c) The Royal College of Ophthalmologists 2019

\section{Introduction}

The presence of silicone oil (SO) droplets in the vitreous, even though frequently considered harmless, has been reported in $0.03-1.7 \%$ of eyes receiving intravitreal injections [1].

The source of these droplets is supposedly the syringes, since they are coated with $\mathrm{SO}$ in order to facilitate the

Gustavo Barreto Melo

gustavobmelo@yahoo.com.br

Hospital de Olhos de Sergipe, Aracaju, SE, Brazil

Federal University of São Paulo, São Paulo, SP, Brazil

Retina Center of Minnesota, Minneapolis, MN, USA

4 Chemical Analysis Laboratory, Center for Chemistry and Manufactured Goods, Institute for Technological Research, São Paulo, Brazil

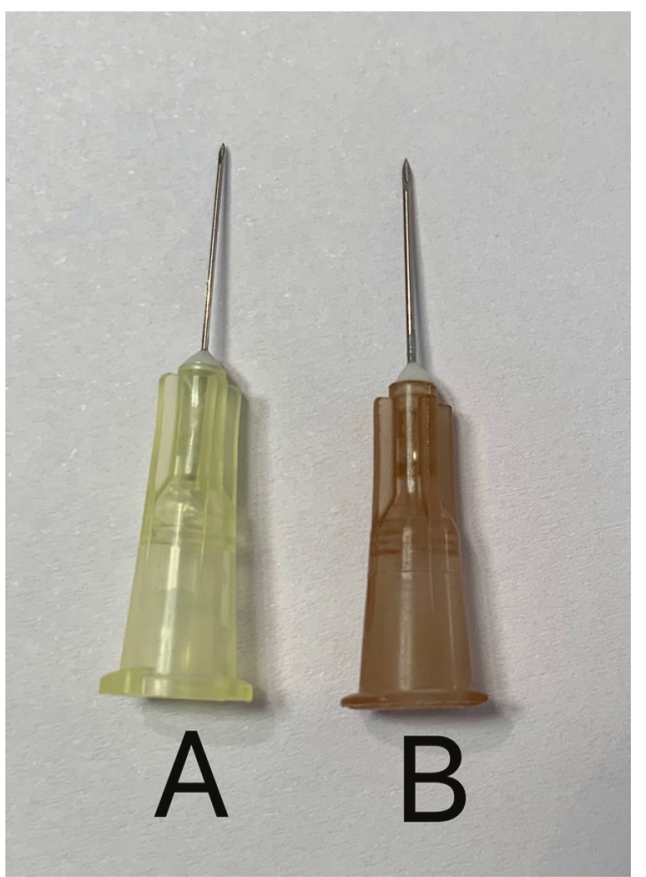

Fig. 1 BD PrecisionGlide needles. a 30-gauge needle; b 26-gauge needle 

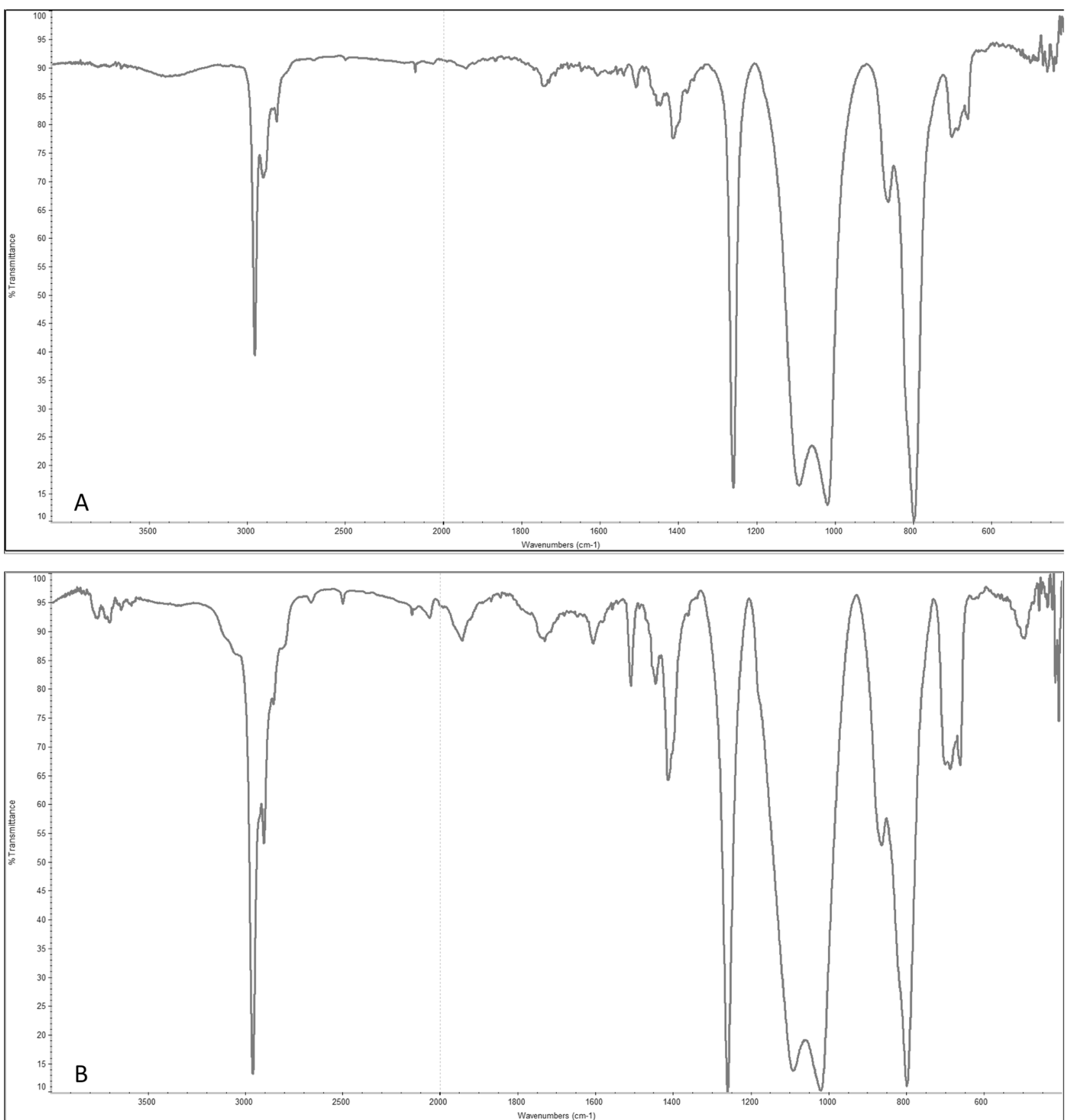

Fig. 2 Fourier-transform infrared spectroscopy showing characteristic bands corresponding to polysiloxane, i.e., silicone oil, in both BD PrecisionGlide needles. a 30-gauge needle; b 26-gauge needle

gliding of the plunger [2]. However, it is uncertain the role the needles might have. Therefore, this study assessed whether two models of needles contained SO.

\section{Materials and methods}

One hundred samples each of two models of needles (Fig. 1) from the same manufacturer were studied: BD PrecisionGlide 26-gauge (Becton-Dickinson, Curitiba, Brazil, lot \# 8234979, ref \# 300110) and 30-gauge needle (lot \# 8165866, ref \# 990193). The metallic part of the needle was removed from the plastic hub. Groups of 50 samples were immersed in $50 \mathrm{~mL}$ of chloroform (99.8\%, Anidrol, São Paulo, Brazil) for $30 \mathrm{~min}$ under ultrasound, $40 \mathrm{kHz}$ (USC-4800, Unique, São Paulo, Brazil). Then the chloroform was evaporated in a water bath (TE-056, Tecnal, São Paulo, Brazil) followed by an oven (Q314M212, Quimis, São Paulo, Brazil) at $105^{\circ} \mathrm{C}$. After cooling, 
the mass of the extract was verified by an analytical scale (AB204-S, Mettler Toledo, Switzerland). The dry extract was assessed by Fourier-transform infrared spectroscopy (Nicolet iS10, Thermo Electron Scientific Instruments, Madison, WI), with band ranging from $4000 \mathrm{~cm}^{-1}$ to $400 \mathrm{~cm}^{-1}$, resolution of $4 \mathrm{~cm}^{-1}$, and 32 scans.

\section{Results}

Analysis of the material indicated the presence of polysiloxane (i.e., SO) in both needles (Fig. 2). The following characteristic bands were found: $2960 \mathrm{~cm}^{-1}$, stretching vibrations of $\mathrm{CH}_{3}$ in $\mathrm{Si}-\mathrm{CH}_{3} ; 1260 \mathrm{~cm}^{-1}$, bending vibrations of $\mathrm{CH}_{3}$ in $\mathrm{Si}-\mathrm{CH}_{3} ; 1090 \mathrm{~cm}^{-1}$ and $1020 \mathrm{~cm}^{-1}$, asymmetric stretching vibrations of $\mathrm{Si}-\mathrm{O}-\mathrm{Si} ; 800 \mathrm{~cm}^{-1}$, stretching vibrations of $\mathrm{Si}-\mathrm{C}$ in $\mathrm{Si}_{-}-\mathrm{CH}_{3}$ [3].

Both 30- and 26-gauge needles contained on average 1 and $1.5 \mathrm{mg}$, respectively, of polysiloxane for every 50 samples, yielding an estimate of 20 and $30 \mu \mathrm{g}$, respectively, per unit.

\section{Discussion}

SO is a lubricant commonly used in medical catheters, syringes, and needles to aid comfort during insertion and control during the delivery of medication. The main concern about $\mathrm{SO}$ in the eye is that it may cause symptomatic floaters. A report from the American Society of Retina Specialists showed that $5.2 \%$ of US retina specialists have performed vitrectomy to remove SO droplets after intravitreal injection of bevacizumab [4]. This is undesirable due to the inherent risks of invasive procedures.

Our suspicion for the presence of SO in needles follows from a separate study where we identified rare SO droplets in fluid ejected from a silicone-free syringe via a BD PrecisionGlide needle (unpublished data). The current analysis confirms that $\mathrm{SO}$ is present in two needle models; in other words, the use of silicone-free syringes does not completely eliminate the risk of SO droplets in the vitreous so long as the needle contains SO.

In conclusion, ophthalmologists should be aware that needles tend to be coated with SO and there is a potential risk of displacing some droplets into the eye.

Funding EyePharma (São Paulo, Brazil) supported this study.

Author contributions All authors conceived and/or designed the work that led to the submission, acquired data, and/or played an important role in interpreting the results, drafted or revised the manuscript, approved the final version, and agreed to be accountable for all aspects of the work.

\section{Compliance with ethical standards}

Conflict of interest The authors declare that they have no conflict of interest.

Publisher's note: Springer Nature remains neutral with regard to jurisdictional claims in published maps and institutional affiliations.

\section{References}

1. Khurana RN, Chang LK, Porco TC. Incidence of presumed silicone oil droplets in the vitreous cavity after intravitreal bevacizumab injection with insulin syringes. JAMA Ophthalmol. 2017;135:800-3.

2. Funke S, Matilainen J, Nalenz H, Bechtold-Peters K, Mahler HC, Friess W. Analysis of thin baked-on silicone layers by FTIR and 3D-laser scanning microscopy. Eur J Pharm Biopharm. 2015;96:304-13. https://doi.org/10.1016/j.ejpb.2015.08.009

3. Chamerski K, Lesniak M, Sitarz M, Stopa M, Filipecki J. An investigation of the effect of silicone oil on polymer intraocular lenses by means of PALS, FT-IR and Raman spectroscopies. Spectrochim Acta A Mol Biomol Spectrosc. 2016;167:96-100. https://doi.org/10.1016/j.saa.2016.05.036

4. Stone TW, editor. ASRS 2018 preferences and trends membership survey. Chicago, IL: American Society of Retina Specialists; 2018. https://www.asrs.org/content/documents/_2018-pat-survey-resultsfor-website.pdf. Accessed 29 Aug 2018. 